\title{
A simple modulation algorithm for inter-phase balancing in CHB Converters
}

\author{
Pablo Montero, and Francisco Gordillo, Member \\ Department of Systems Engineering and Automation \\ University of Seville, Spain
}

\begin{abstract}
This paper presents a simple modulation algorithm which accomplishes the voltage balance across the capacitors of a Cascaded H-bridge $(\mathrm{CHB})$ converter using zero voltage injection. CHB converters are widely used in renewable energy production, specially in solar plants due to its modularity. This advantage can also become a trouble when the power generated by the solar cell or consumed by a load is not equal among the three phases, leading to an unbalanced situation. A well-known technique consists in injecting a zero sequence voltage according to the power distribution. A simple modulation algorithm is presented in this paper including a mathematical proof. The proposed algorithm has been tested by simulation with a fivelevel CHB converter. At the end of the paper, a modification is introduced to improve the converter efficiency.
\end{abstract}

\section{INTRODUCTION}

$\mathbf{M}$ ULTILEVEL converters have found a wide range of applications from power converter for renewable energy integration to electric vehicle uses as they have been growing in popularity [1]. Nowadays Several multilevel converters topologies have been presented and the number of levels it can be reached seems never ending. Generally, a multilevel converter uses several capacitors to provide differents levels of voltage. This fact lets to use power semiconductors of lower operating voltage, reducing losses and allowing a power semiconductor device to be used in a higher voltage application. However, having more than one capacitor leads to the necessity of controlling the voltage difference among them, increasing the control complexity. Because of this, a external control is usually used to balance the different voltage capacitors.

Among the different multilevel converter topologies, this papers focus on the Cascaded H-bridge (CHB) converter [2]. A CHB converter is composed of a string of $\mathrm{H}$-bridges, allowing every cell to set null voltage, the direct or the reverse voltage drop of the capacitor between its terminals. Due to the modularity that $\mathrm{CHB}$ converter offers, different string of solar panels can be connected to each H-bridge cell, being possible to reach the optimum power point individually [3]. If the number of levels of the three-phase converter is more than 3 then there are more than one H-bridge per phase, hence the unbalance issue not only appears as an inter-phase problem but also as an in-phase one. In [4] the in-phase balance is analyzed and a solution is presented for a single-phase five-level CHB converter from a power distribution perspective.

There are many results in the literature which focus on how to achieve the dc-link voltage balance for the cells of different phases for a three-phase system [5]. Therefore, this paper would focus on balancing the total dc-link voltage using zero voltage injection, which can only be used in a threephase system [6], [7]. This technique consists in adding a zero voltage sequence, which has no effect on the current tracking, to the reference voltage obtained by the current control allowing to charge or discharge the capacitors on a specific phase by selecting the phase and the amplitude of it.

In [8] a zero sequence voltage injection and a negative sequence voltage injection are presented together with its control scheme as a methodology to solve the unbalance. In [9] a zero sequence voltage injection is included in a phase-shift PWM and a CHB converter is tested with this modulation technique. In [10] the inphase balance is presented in an one-cycle control in addition to the tracking of the zero component calculated to correct the unbalance. In this paper, a 3-phase five-level CHB converter is analyzed under differents loads for each phase and a voltage balance algorithm is presented. The main advantage of the presented algorithm is its simplicity compared to the solutions cited previously and the guarantee it works under the conditions obtained by the mathematical proof.

To evaluate the performance of the converter and the algorithm it is assumed that it is connected to a balanced symmetrical grid. In Section II, the model of the five-level converter is discussed together with the proposed balancing algorithm. Section III presents the mathematical proof resulting in the conditions under which the voltage balance is guaranteed. Afterwards, in section IV a modification in the algorithm which reduce the voltage ripple of the neutral point is presented. Section V shows the results of the simulation and the value of the simulation parameters. Finally, a conclusion about the algorithm and future work is provided.

\section{Problem STATEMEnT AND CONTROL LAW MOTIVATION}

In this section a five-level CHB Converter (Fig. 1) will be presented with the equations of its model. This system is composed by two H-bridge cells per phase and they are connected to the grid $U_{a b c}$ through an inductor $L$. Variables $I_{a}, I_{b}$ and $I_{c}$ are the phase currents which are defined positive as inputs to the converter. Inside each cell there is a capacitor denoted as $C_{i j}$ where $i \in\{a, b, c\}$ and $j \in\{1,2\}$ according to the phase and the position of the capacitor. Each cell has a parallel constant power load and the current through the load is named $I_{d c i j}$ with the same criteria than the capacitors. 


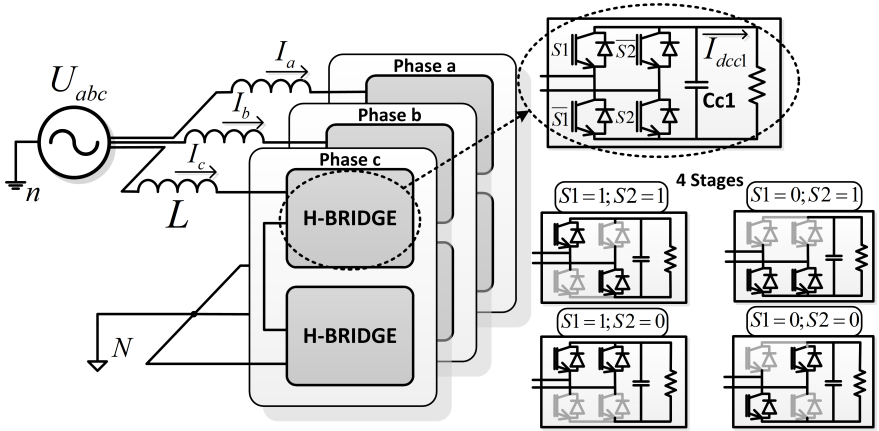

Fig. 1. A five level CHB grid-connected converter

There are four switching devices in every H-bridge cell. Considering the switching-on signal of two of them complementary there are 4 possible stages, which are also depicted in Fig. 1. Thus, a five-level CHB converter yields 16 possible conmutation stages per phase which are shown in Table I. In this table the switching stages are classified according to the switching-on signals - firstly the $S 1$ and $S 2$ signal of the top cell $\left(S 1^{T} S 2^{T}\right)$ and then, the same signals of the bottom cell $\left(S 1^{B} S 2^{B}\right)$. Depending on the value of the Voltage output of each stage, a state number is associated to it. Within the same state several redundant stages exist, thus a substate number is associated to each of them.

TABLE I

SWITCHING STAGES PER PHASE OF A FIVE-LEVEL CHB CONVERTER

\begin{tabular}{|c|c|c|}
\hline Switching - on Signals & State-Substate & Voltage Output \\
\hline 1111 & 1 & $V_{C i 1}+V_{C i 2}$ \\
\hline 0111 & 21 & $V_{C i 2}$ \\
\hline 1011 & 22 & \\
\hline 1101 & 23 & $V_{C i 1}$ \\
\hline 1110 & 24 & \\
\hline 0101 & 31 & 0 \\
\hline 1001 & 32 & \\
\hline 0110 & 33 & \\
\hline 1010 & 34 & \\
\hline 1100 & 35 & \\
\hline 0011 & 36 & \\
\hline 0100 & $\overline{41}$ & $-V_{C i 2}$ \\
\hline 1000 & 42 & \\
\hline 0010 & 43 & $-V_{C i 1}$ \\
\hline 0001 & 44 & \\
\hline 0000 & 5 & $\overline{-}-V_{C i 1}-V_{C i 2}$ \\
\hline
\end{tabular}

Considering the stages displayed and the capacitor equation:

$$
\begin{aligned}
C \frac{d V_{C i 1}}{d t} & =I_{i}\left(f_{1}+f_{23}+f_{24}+f_{35}-f_{36}\right. \\
& \left.-f_{43}-f_{44}-f_{5}\right)-I_{d c i 1} \\
C \frac{d V_{C i 2}}{d t} & =I_{i}\left(f_{1}+f_{21}+f_{22}+f_{36}-f_{35}\right. \\
& \left.-f_{41}-f_{42}-f_{5}\right)-I_{d c i 2}
\end{aligned}
$$

Where $i \in\{a, b, c\}$ and $f_{x y}$ is the unitary percentage of a switching cycle corresponding to the case where the two cells were in the state $x$ and substate $y$. Every state $(x)$ consists of a number of substates (y), except for the states 1 and 5 which has no substate and the $y$ annotation is not needed. The signal $f_{x}$ represents the unitary percentage of the whole $x$ state. According to this definition the following constraints have to be fullfilled:

$$
\sum_{y=1}^{4} f_{x y}=f_{x}, \quad x \in\{2,4\} ; \quad \sum_{y=1}^{6} f_{3 y}=f_{3} ; \quad \sum_{x=1}^{5} f_{x}=1
$$

Taking the phase average dc-link values and adding up the dc currents, the following two variables are defined:

$$
\begin{aligned}
V_{C i} & =\frac{V_{C i 1}+V_{C i 2}}{2} \\
I_{d c i} & =I_{d c i 1}+I_{d c i 2}
\end{aligned}
$$

where $i \in\{a, b, c\}$. Adding (1) and (2) and using (3) and (4) yields:

$$
C \frac{d V_{C i}}{d t}=\frac{I_{i}}{2}\left(2 f_{1}+f_{2}-f_{4}-2 f_{5}\right)-\frac{I_{d c i}}{2}
$$

where $\left(2 f_{1}+f_{2}-f_{4}-2 f_{5}\right)$ is equal to the output waveform $m_{i} \in[-2,2]$, which is set by the current control. With this definition, eq. (5) can be written as:

$$
C \frac{d V_{C i}}{d t}=\frac{I_{i} m_{i}}{2}-\frac{I_{d c i}}{2}
$$

To characterize the interphase issue two error signals has to be defined:

$$
\begin{aligned}
& V_{d 1}=V_{d c}-V_{C a} \\
& V_{d 2}=V_{d c}-V_{C b}
\end{aligned}
$$

where $V_{d c}=\frac{V_{C a}+V_{C b}+V_{C c}}{3}$. Thus, derivating (7) and (8) and combining them with (6) yields:

$$
\begin{aligned}
& C \frac{d V_{d 1}}{d t}=\frac{1}{6}\left(-2 I_{a} m_{a}+I_{b} m_{b}+I_{c} m_{c}+\gamma_{1}\right) \\
& C \frac{d V_{d 2}}{d t}=\frac{1}{6}\left(I_{a} m_{a}-2 I_{b} m_{b}+I_{c} m_{c}+\gamma_{2}\right)
\end{aligned}
$$

where $\gamma_{1}$ is equal to $2 I_{d c a}-I_{d c b}-I_{d c c}$ and $\gamma_{2}$ is equal to $-I_{d c a}+2 I_{d c b}-I_{d c c}$. By adding a new component $x$ to the signals $m_{a}, m_{b}$ and $m_{c}$ in (9)-(10) and considering $I_{a}+I_{b}+$ $I_{c}=0$ results in:

$$
\begin{aligned}
& \dot{V_{d 1}}=\frac{1}{6 C}\left(-2 I_{a} m_{a}+I_{b} m_{b}+I_{c} m_{c}+\gamma_{1}-3 I_{a} x\right) \\
& \dot{V_{d 2}}=\frac{1}{6 C}\left(I_{a} m_{a}-2 I_{b} m_{b}+I_{c} m_{c}+\gamma_{2}-3 I_{b} x\right)
\end{aligned}
$$

The addition of this new signal $x$ equals a zero component injection given the fact that the value is the same for the three phases. To determine what is the value of $x$ that reduces the error signals $V_{d 1}$ and $V_{d 2}$, a Lyapunov function candidate is defined:

$$
W=\left|V_{d 1}\right|+\left|V_{d 2}\right|
$$

The target is to reduce the value of $W$ as much as possible, which equals to make $\dot{W}$ the most negative value achievable. Special attention should be paid to (13) as it is not differentiable around $V_{d 1}=0$ and $V_{d 2}=0$. Thus, differentiating (13) assuming constants signs:

$$
\dot{W}=\operatorname{sign}\left(V_{d 1}\right) \dot{V}_{d 1}+\operatorname{sign}\left(V_{d 2}\right) \dot{V}_{d 2}
$$


$\dot{W} \cdot 6 C=\operatorname{sign}\left(V_{d 1}\right)\left(-2 I_{a} m_{a}+I_{b} m_{b}+I_{c} m_{c}+\gamma_{1}\right)+\operatorname{sign}\left(V_{d 2}\right)\left(I_{a} m_{a}-2 I_{b} m_{b}+I_{c} m_{c}+\gamma_{2}\right)-3 x\left(\operatorname{sign}\left(V_{d 1}\right) I_{a}+\operatorname{sign}\left(V_{d 2}\right) I_{b}\right)$

Combining (11) and (12) into (14), (15) is obtained. In order to avoid overmodulation, the highest and lowest value achievable for $x$ is limited by $\left(m_{i}+x\right) \in[-2,2]$. Inspired by the last term of (15) and considering this limitation, the resulting algorithm would conclude as:

$$
\begin{aligned}
\text { if } & \left(\operatorname{sign}\left(V_{d 1}\right) I_{a}+\operatorname{sign}\left(V_{d 2}\right) I_{b}\right) \leq 0 \\
\text { then } & x=-2-\min \left(m_{a}, m_{b}, m_{c}\right) \\
\text { else } & x=2-\max \left(m_{a}, m_{b}, m_{c}\right)
\end{aligned}
$$

This algorithm can be used in any control methods which involves the output waveform $\left(m_{i}\right)$ computation. Once $m_{i}$ is computed as usual, the $x$ value is calculated using (16) and added afterwards. The algorithm (16) relies on its simplicity as no further computation is required.

\section{MAIN RESULT}

Theorem 1. Consider the voltage differences dynamics (11)(12) with the output waveforms given by the current control and the value of $x$ given by (16). If $0.26 P_{t}<P_{c}<0.406 P_{t}$ and $P_{t}(0.874-2 p)<P_{c}<P_{t}(1.1261-2 p)-$ where $P_{t}$ is equal to the system input power; $P_{a}, P_{b}$ and $P_{c}$ is equal to the power demanded by the phase $a, b$ and $c$ respectively; and $p=\frac{P_{b}}{P_{t}}-$ then, the voltage differences $V_{d 1}$ and $V_{d 2}$ tend to band around zero.

Proof. it will be proved that $\Delta W$ is always negative when evaluated at the grid frequency rate. This statement is only valid when the absolute values of the error signals at the beginning of the evaluated grid period is high enough to prevent them from changing their sign for the whole grid period.

\section{A. Assumptions}

To begin with, it is assumed that the total voltage and current controllers have reached the steady state, thus the input power is equal to the load demand and the currents and output waveform signals are senoidals. It is also assumed that there is no exchange of reactive power between the grid and the system. The balance of the cells in the same phase is not the aim of this paper, so the loads of each cell within the same phase are assumed equal.

$$
\begin{aligned}
I_{a}=A \cos (w t) \quad m_{a}=\frac{V_{r e f}}{V_{C a}} \cos (w t-\phi) \\
I_{b}=A \cos \left(w t-\frac{2 \pi}{3}\right) \quad m_{b}=\frac{V_{r e f}}{V_{C b}} \cos \left(w t-\frac{2 \pi}{3}-\phi\right) \\
I_{c}=A \cos \left(w t+\frac{2 \pi}{3}\right) \quad m_{c}=\frac{V_{r e f}}{V_{C c}} \cos \left(w t+\frac{2 \pi}{3}-\phi\right)
\end{aligned}
$$

Where $A$ is the current amplitude, $V_{\text {ref }}$ is the amplitude of the reference voltage and $\phi$ is the constant phase angle difference between the output waveform and the grid voltage. From now on, $V_{r e f_{a}}=V_{\text {ref }} \cos (w t-\phi), V_{\text {ref }}=$
$V_{\text {ref }} \cos \left(w t-\frac{2 \pi}{3}-\phi\right)$ and $V_{\text {ref }}=V_{\text {ref }} \cos \left(w t+\frac{2 \pi}{3}-\phi\right)$. It is also assumed that the loads absorb a constant amount of power (18).

$$
I_{d c a}=\frac{P_{a}}{V_{C a}} ; \quad I_{d c b}=\frac{P_{b}}{V_{C b}} ; \quad I_{d c c}=\frac{P_{c}}{V_{C c}}
$$

\section{B. Case analysis}

Four possible cases are obtained when assuming a constant sign of the error signals. By introducing (17) and integrating the possible cases in (15) over a grid period, four inequalities are obtained: (19) and (20); which have to be accomplished in order to succeed in proving the good behaviour of the algorithm. To summarize, two integrals $-H$ for the left side of the inequality and $F(x)$ for the right side of the inequality - have to be evaluated for each case.

$$
\int_{0}^{T} H d \omega t<\int_{0}^{T} F(x) d \omega t
$$

1) Lower bound for $F$ : In this subsection, a lower bound for $F$ is found in order to consider the worst scenario for the achievement of (21).

Considering the behaviour of $x$ according to the sign of the error signals and the current expressions, the minimum value of the integral can be calculated, proportional to $A$, depending on the value of $\phi$. The evaluation of the two cases of $\int_{0}^{T} F(x) d \omega t$ in (19) and also in (20) results in the same value due to the bahaviour of $x$.

Finally, evaluating $\int_{0}^{T} F(x) d \omega t$ with every possible value of $\phi$, Fig. 2 is obtained. Hence, the lowest value are directly read from it.

For the two cases of (19) these values correspond to (22) and for the two cases of (20) these values correspond to (23):

$$
\begin{aligned}
& \int_{0}^{T} \pm 3 x\left(I_{a}+I_{b}\right) d \omega t>4.11 A \\
& \int_{0}^{T} \pm 3 x\left(I_{a}-I_{b}\right) d \omega t>7.13 A
\end{aligned}
$$

2) Upper bond for $H$ : In order to compute an upper bound for $\int_{0}^{T} H d \omega t$ some aproximations will be made, always from a conservative view.

Taking into account that the input power is equal to the power demanded by all the loads, it is evident that the signs of the error signals show if the power demanded by a phase $(a, b$ or $c)$ is over or under the third part of the incoming power. For example, consider the case $V_{d 1}>0$ and $V_{d 2}=0$, this would mean that $V_{C a}$ is under the average level $\left(V_{d c}\right)$ while $V_{C b}$ is equal to the average level and thus $V_{C c}$ is over the average level.

As the output waveform signals $m_{i}$ for $i=\{a, b, c$,$\} are$ equal but out of phase -similar to the phase currents- the 


$$
\begin{aligned}
& V_{d 1} \gtrless 0 ; V_{d 2} \gtrless 0: \int_{0}^{T}\left( \pm \frac{1}{V_{C a}}\left(P_{a}-I_{a} V_{r e f_{a}}\right) \pm \frac{1}{V_{C b}}\left(P_{b}-I_{b} V_{\text {ref }}\right) \pm \frac{2}{V_{C c}}\left(-P_{c}+I_{c} V_{r e f_{c}}\right)\right) d \omega t<\int_{0}^{T} \pm 3 x\left(I_{a}+I_{b}\right) d \omega t \\
& V_{d 1} \gtrless 0 ; V_{d 2} \lessgtr 0: \int_{0}^{T}\left( \pm \frac{3}{V_{C a}}\left(P_{a}-I_{a} V_{r e f_{a}}\right) \pm \frac{3}{V_{C b}}\left(I_{b} V_{r e f_{b}}-P_{b}\right)\right) d \omega t<\int_{0}^{T} \pm 3 x\left(I_{a}-I_{b}\right) d \omega t
\end{aligned}
$$

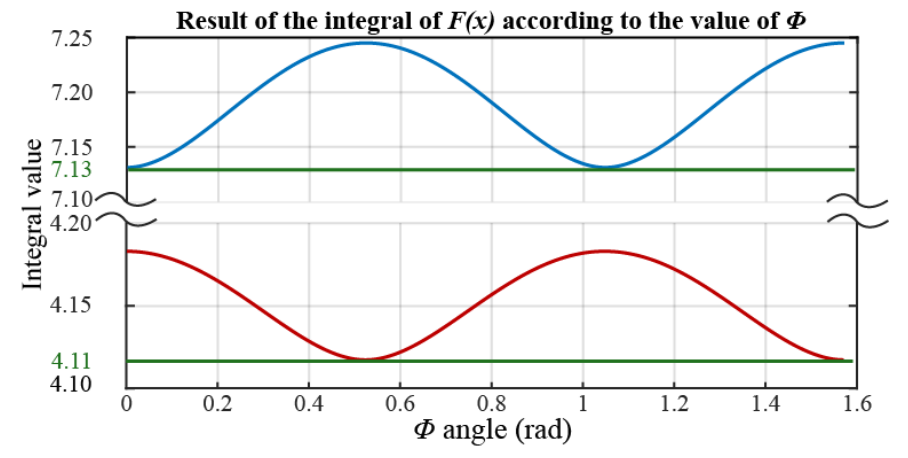

Fig. 2. Values for both groups of $\int_{0}^{T} F(x)$ depending on the value of $\phi$

power supplied to each phase is equal among them, hence $\int_{0}^{T} I_{i} V_{\text {ref }_{i}} d \omega t=P_{t} / 3$ for $i \in\{a, b, c\}$. If the loads of a phase demands a different amount of power then its average capacitor voltage level $\left(V_{C i}\right)$ would change, increasing in the case of demanding less power or decreasing in the case of demanding more power. Therefore, in the case exemplified, the phase $a$ load is demanding more power than the third part of the incoming power while the phase $\mathrm{c}$ load is demanding less power.

By applying the same logic with the four possible cases of error signals signs, Table II is obtained. When the error signal

TABLE II

RELATION BETWEEN THE THIRD PART OF THE INPUT POWER AND THE POWER DEMANDED BY THE LOADS OF EACH PHASE, DETERMINED BY THE SIGNS OF THE ERROR SIGNALS

\begin{tabular}{ccccc}
\hline$V_{d 1}$ & $V_{d 2}$ & $P_{a}$ & $P_{b}$ & $P_{c}$ \\
\hline$>0$ & $>0$ & $>$ & $>$ & $<$ \\
\hline$>0$ & $<0$ & $>$ & $<$ & \\
\hline$<0$ & $>0$ & $<$ & $>$ & \\
\hline$<0$ & $<0$ & $<$ & $<$ & $>$
\end{tabular}

signs are different, the power demanded by phase $c$ can not be determined only by the signs but it is not necessary as it does not appear in (20).

The conclusion is that the upper bound of $\int_{0}^{T} H d \omega t$ can be obtained maximizing all the terms that appear in it, since they are all positive under its own case. In order to bound them, it is considered that the average capacitor voltage level of each phase can not underpass a minimum level of voltage as a conservative measure; since they appear in the denominator of (19) and (20). This value is equal to that which allows to modulate a whole grid wave without overmodulating. Denoting the RMS voltage value of the grid as $V_{g}^{R M S}$, the minimum value allowed will be $V_{C_{i_{m i n}}}=V_{g}^{R M S} / \sqrt{2}$. By equalling this value to $V_{C a}, V_{C b}$ and $V_{C c}$ into (19) and (20):

$$
\begin{aligned}
& V_{d 1} \gtrless 0 ; V_{d 2} \gtrless 0: \int_{0}^{T} \pm \frac{1}{V_{C_{i_{\text {min }}}}}\left(P_{a}+P_{b}-2 P_{c}\right) d \omega t \\
& V_{d 1} \gtrless 0 ; V_{d 2} \lessgtr 0: \int_{0}^{T} \pm \frac{3}{V_{C_{i_{\text {min }}}}}\left(P_{a}-P_{b}\right) d \omega t
\end{aligned}
$$

Applying the definition of the total power $P_{t}=P_{a}+P_{b}+P_{c}$ to (24) and the variable $p=P_{t} / P_{b}$ to (25):

$$
\begin{array}{ll}
V_{d 1} \gtrless 0 ; \quad V_{d 2} \gtrless 0: & \pm \frac{2 \pi}{V_{C i_{\text {min }}}}\left(P_{t}-3 P_{c}\right) \\
V_{d 1} \gtrless 0 ; \quad V_{d 2} \lessgtr 0: & \pm \frac{6 \pi}{V_{C i_{\text {min }}}}\left(P_{t}(1-2 p)-P_{c}\right)
\end{array}
$$

3) Comparision between integrals: By comparing the values of the integrals obtained: (26) with (22), (27) with (23); and using the definition of power in an AC System where there is no reactive power involved $P_{t}=3 \frac{A}{\sqrt{2}} V_{g}^{R M S}$, which equals to $A V_{C_{i_{\text {min }}}}=\frac{P_{t}}{3}$, the restrictions for each case are obtained:

$$
\begin{array}{lll}
V_{d 1}>0 ; & V_{d 2}>0: & P_{c}>0.26 P_{t} \\
V_{d 1}>0 ; & V_{d 2}<0: & P_{c}>P_{t}(0.874-2 p) \\
V_{d 1}<0 ; & V_{d 2}>0: & P_{c}<P_{t}(1.1261-2 p) \\
V_{d 1}<0 ; & V_{d 2}<0: & P_{c}<0.406 P_{t}
\end{array}
$$

These restrictions result in a power region depicted in Fig. 3 where the algorithm is capable of correcting the unbalance.

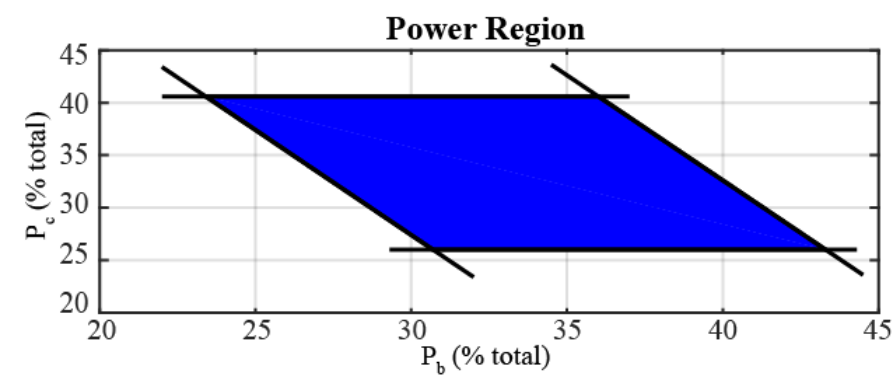

Fig. 3. Power region where the algorithm has been proven mathematically

\section{IMPROVING THE NEUTRAL POINT VOLTAGE RIPPLE}

In order to improve the system efficiency in a solar system, the voltage ripple of the neutral point (depicted as $\mathrm{N}$ in Fig. 1) has to be reduced. A current leakage exists between the neutral point and the ground that might be modelled as a capacitor, thus the bigger the voltage ripple is the greater the losses are. 
The previous algorithm always applies the maximum absolute value for $x$, thus in steady state, where the error signals signs may change within a switching period, $x$ can be switched from his maximum value to his minimum value from a switching instant to the next one. This would lead to a high ripple of the voltage drop between the neutral point and the ground $\left(V_{N n}\right)$. In order to reduce it, the switching has to be smoother by restricting the algorithm behaviour. A new variable $K_{x}$ is defined for this porpuse, whose aim is to reduce the value of the $x$ applied. The variable $K_{x}$ would multiply $x$ and its value would be reduced progressively by a proportional control as the Lyapunov function $(W)$ gets closer to the steady state:

$$
\begin{gathered}
x^{\prime}=K_{x} \cdot x \\
K_{x}=\operatorname{sat}\left(K_{p}(W-\widetilde{W})\right) \quad K_{x} \in[0,1]
\end{gathered}
$$

where 'sat' is the standard saturation between zero and one, $x^{\prime}$ is the new applicable value of $x, K_{p}$ is the control parameter of the proportional control, $W$ is the value of the Lyapunov function defined in (13) and $\widetilde{W}$ is the reference that the modified algorithm will make the Lyapunov function to reach in the steady state. The modified algorithm would no longer try to reach the zero value of the Lyapunov function but the value indicated by $\widetilde{W}$.

Whenever the value of $K_{x}$ is equal to 1 , the modified algorithm behaviour is identical to the original one, thus the signals error would be reduced provided that the system conditions are inside the power region. The value of $K_{x}$ starts declining when $W$ reach a specific value $\left(W_{\text {band }}\right)$ given by:

$$
K_{p}\left(W_{\text {band }}-\widetilde{W}\right)=1 \rightarrow W_{\text {band }}=\frac{1}{K_{p}}+\widetilde{W}
$$

Therefore, the modified algorithm will make $W$ to be below $W_{\text {band }}$, since the original algorithm is applied when $W$ is over $W_{\text {band }}$.

\section{Simulation Results}

The parameters of the simulation are shown in the table III. Notice that the power of each phase are out of the power region, which means that the algorithm is able to work in an extended region in a real application and the region depicted in Fig. 3 is a conservative result.

TABLE III

SIMULATION PARAMETERS

\begin{tabular}{cc}
\hline Parameter & Value \\
\hline \hline$V_{d c_{r e f}}$ & $300 \mathrm{~V}$ \\
\hline$P_{a}$ & $7000 \mathrm{~W}$ \\
\hline$P_{b}$ & $5000 \mathrm{~W}$ \\
\hline$P_{c}$ & $8000 \mathrm{~W}$ \\
\hline$L$ & $3.3 \mathrm{mH}$ \\
\hline$V_{g}$ & $220 V_{r m s}$ \\
\hline$C_{d c_{\text {link }}}$ & $2000 \mu \mathrm{f}$ \\
\hline
\end{tabular}

The Fig. 4 shows the diagram block of the implemented control. It consists in a voltage controller, which provide a reference power $\left(P_{r e f}\right)$ from the desired dc-link voltage $\left(\tilde{V}_{d c}\right)$ and the average dc-link voltage $\left(V_{d c}\right)$, that is transformed into the reference currents in $d q$ axis $\left(\tilde{I}_{d}\right.$ and $\left.\tilde{I}_{q}\right)$. This references are compared with the real ones in the current control scheme, resulting in two voltage references $\left(\tilde{V}_{d}\right.$ and $\left.\tilde{V}_{q}\right)$, that are backtransformed to $a b c$ axis $\left(V_{r e f_{a}}, V_{r e f_{b}}\right.$ and $\left.V_{r e f_{c}}\right)$ and divided by the average dc-link voltage of his phase branch $\left(V_{C_{a}}, V_{C_{b}}\right.$ and $\left.V_{C_{c}}\right)$ to obtain the output waveforms $\left(m_{a}, m_{b}\right.$ and $\left.m_{c}\right)$. Next, (16) is applied and the modified output waveforms are obtained $\left(m_{a}^{\prime}, m_{b}^{\prime}\right.$ and $\left.m_{c}^{\prime}\right)$. The signals $\omega$ and $\omega t$ are the grid frequency $(\mathrm{rad} / \mathrm{s})$ and the grid angle $(\mathrm{rad})$ respectively. $Q_{r e f}$ is set to 0 . This control is deeper explained in [11].

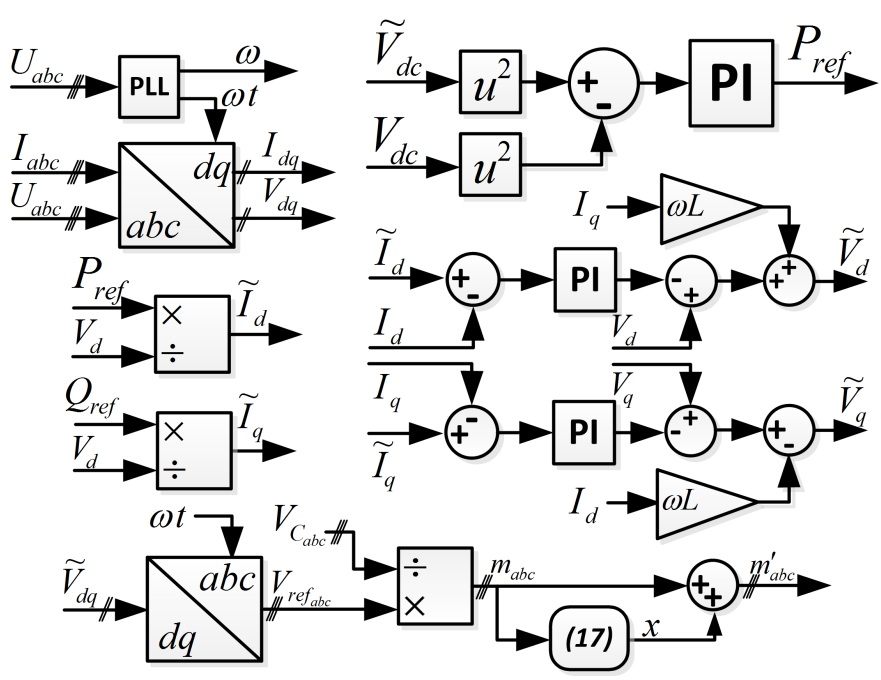

Fig. 4. Block diagram of the control used

The sequence this simulation follows starts with a preload stage with no loads. After it, the voltage and current controls are activated, although the value of $x$ is set to zero and no loads are still connected. At $t=0.35 s$ the constant power loads are activated and at $t=0.4 \mathrm{~s}$ the balancing algorithm is activated.

In Fig. 5 the evolution of the DC voltages of each phase are depicted along with the error signals $V_{d 1}$ and $V_{d 2}$ for both the original and the modified algorithm. It can be seen that there is a tendency to unbalance the capacitors from the moment the loads are activated $(t=0.35 \mathrm{~s})$. This tendency is supressed and corrected from the moment the algorithm is activated $(t=$ $0.5 \mathrm{~s})$. In the modified algorithm simulation, the parameter $\widetilde{W}$ is equal to 35 and $K_{p}=0.1$. As can be seen in Fig. 6 , the inclusion of the algorithm does not take a remarkable effect on the reference currents tracking. In Fig. 7 the ripple of the voltage $V_{N n}$ is compared in both cases through a Fourier analysis. It can be seen a considerable harmonics reduction with the modified algorithm.

\section{CONCLUSiON}

The algorithm presented in this paper is a new and a very simple way of using the zero current injection to balance the capacitors in a CHB converter. A modification is introduced in order to make it more appealing to Solar system integration. Despite of making the mathematical analysis for power 


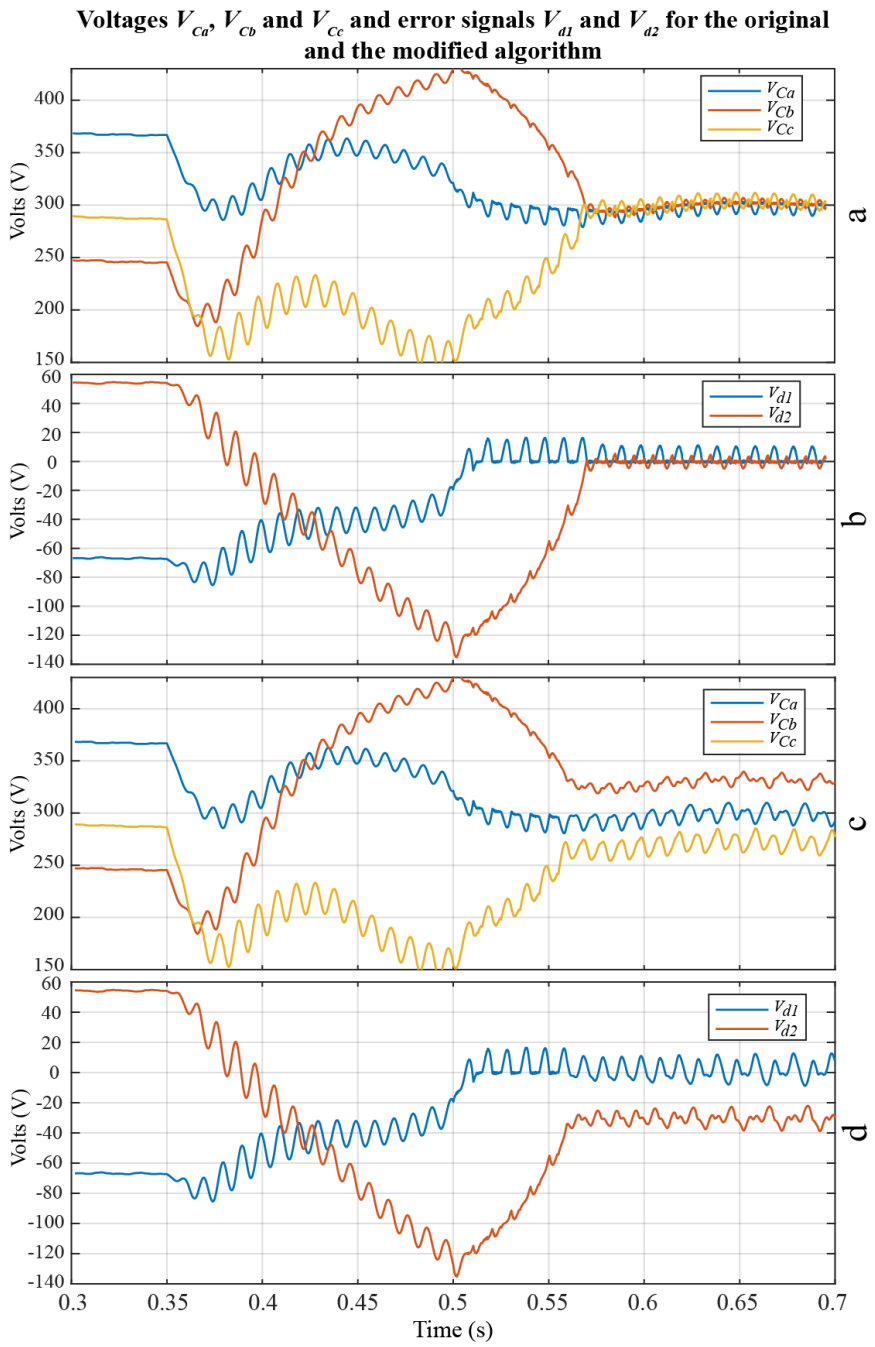

Fig. 5. (a) Signals $V_{C a}, V_{C b}$ and $V_{C c}$ with the original algorithm; (b) Error signals $V_{d 1}$ and $V_{d 2}$ with the original algorithm; (c) Signals $V_{C a}, V_{C b}$ and $V_{C c}$ with the modified algorithm; (d) Error signals $V_{d 1}$ and $V_{d 2}$ with the modified algorithm

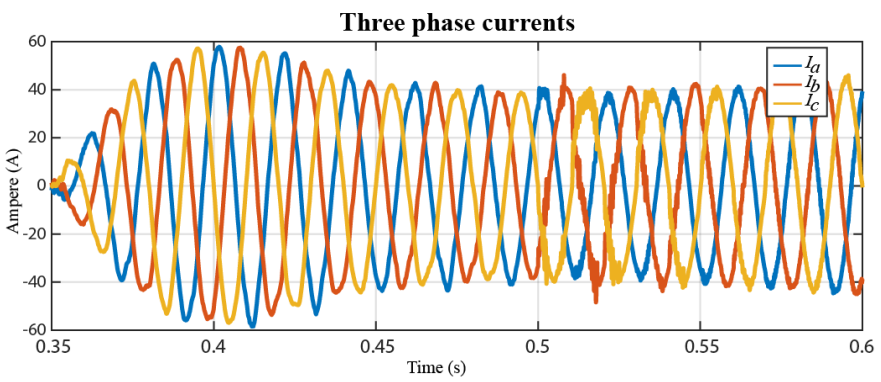

Fig. 6. Evolution of the phase currents in simulation

consuming loads, the analysis can be replicated with power generating loads like solar panels.

As future work, a conmutation reduction could be considered given the fact that this algorithm is focused on balancing the capacitors. This strategy opens a window to new performance-improving strategies along with a basic solution to the unbalance issue of CHB converters that could leads to more complex, more sophisticated algorithm taking advantage

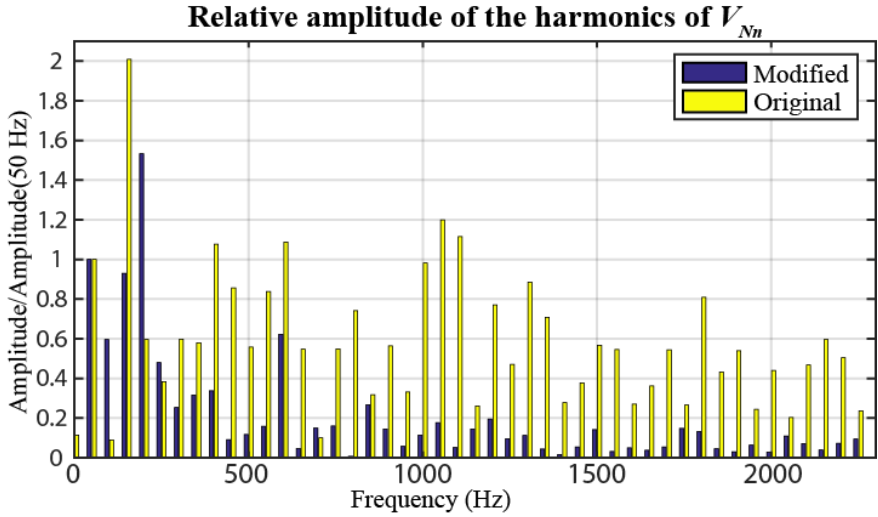

Fig. 7. Comparative in the $V_{N n}$ harmonics amplitude when applying or not the modified algorithm

of the analysis this paper has made.

\section{ACKNOWLEDGMENT}

This work has been funded under grants MINECO-FEDER DPI2013-41891-R and DPI2016-75294-C2-1-R.

\section{REFERENCES}

[1] L. G. Franquelo, J. Rodriguez, J. I. Leon, S. Kouro, R. Portillo, and M. A. M. Prats, "The age of multilevel converters arrives," IEEE Industrial Electronics Magazine, vol. 2, no. 2, pp. 28-39, June 2008.

[2] K. Corzine and Y. Familiant, "A new cascaded multilevel h-bridge drive," IEEE Transactions on Power Electronics, vol. 17, no. 1, pp. 125-131, Jan 2002.

[3] J. N. Ganesh, K. Viswanathan, R. Naik, and S. El-Barbari, "Cascaded hbridge control for pv application," in Power Electronics and Applications (EPE'15 ECCE-Europe), 2015 17th European Conference on, Sept 2015, pp. $1-5$.

[4] S. Vazquez, J. I. Leon, J. M. Carrasco, L. G. Franquelo, E. Galvan, M. Reyes, J. A. Sanchez, and E. Dominguez, "Analysis of the power balance in the cells of a multilevel cascaded h-bridge converter," IEEE Transactions on Industrial Electronics, vol. 57, no. 7, pp. 2287-2296, July 2010

[5] M. Shahabadini, H. Iman-Eini, and S. Afsharnia, "Using auxiliary signals as a simple method for balancing dc bus voltages in cascaded h- bridge converters," in The 6th Power Electronics, Drive Systems Technologies Conference (PEDSTC2015), Feb 2015, pp. 358-362.

[6] N. Jayaram, P. Agarwal, and S. Das, "A three phase five level cascaded h-bridge rectifier with zero current injection scheme," in 2012 IEEE International Conference on Power Electronics, Drives and Energy Systems (PEDES), Dec 2012, pp. 1-7.

[7] P. Palanivel and S. S. Dash, "Control of three phase cascaded multilevel inverter using various novel multicarrier pulse width modulation techniques," in TENCON 2010 - 2010 IEEE Region 10 Conference, Nov 2010, pp. 59-64.

[8] X. Li, H. Geng, G. Yang, R. Li, and C. Zhang, "Comparison of different dc-link voltage balancing methods for cascade h-bridge multilevel converter," in 2014 17th International Conference on Electrical Machines and Systems (ICEMS), Oct 2014, pp. 2465-2470.

[9] M. Zygmanowski, B. Grzesik, and J. Michalak, "Power conditioning system with cascaded h-bridge multilevel converter - dc-link voltage balancing method," in Proceedings of the 2011 14th European Conference on Power Electronics and Applications, Aug 2011, pp. 1-10.

[10] S. Ouyang, J. Liu, X. Wang, and F. Meng, "One-cycle control of a delta-connected cascade h bridge rectifier," in 2014 International Power Electronics and Application Conference and Exposition, Nov 2014, pp. 870-874.

[11] C. Wan, G. Yang, W. Wu, and W. Zhang, "An active and reactive power regulating system based on a cascaded-converter," in The 2nd International Symposium on Power Electronics for Distributed Generation Systems, June 2010, pp. 453-458. 